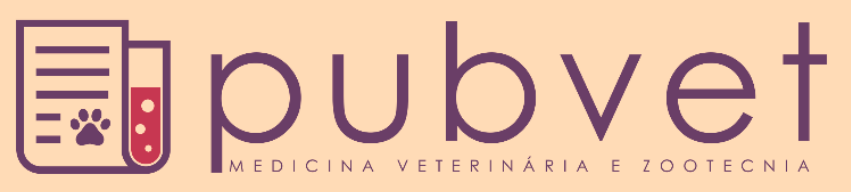

https://doi.org/10.31533/pubvet.v15n08a897.1-5

\title{
Análise comparativa entre glicosímetros portáteis e método laboratorial para a dosagem de glicemia em bovinos
}

\author{
Aline Amaral de Morais $^{1 *} \bullet$, Pricilla Pozzatti ${ }^{2} \bullet$, Carlos Eduardo Nogueira Martins ${ }^{30}$ (D) \\ ${ }^{1}$ Acadêmica de Medicina Veterinária do Instituto Federal Catarinense, Departamento de Patologia Clínica Veterinária. Araquari-SC Brasil. \\ ${ }^{2}$ Professora do Instituto Federal Catarinense, Departamento de Patologia Clínica Veterinária. Araquari-SC Brasil. \\ ${ }^{3}$ Professor do Instituto Federal Catarinense, Departamento de estatística e zootecnia. Araquari-SC Brasil. \\ *Autor para correspondência, E-mail: aline_amaral_morais@hotmail.com
}

\begin{abstract}
Resumo. A glicose é considerada uma importante fonte de energia para as células, crescimento fetal e produção de leite em ruminantes. Algumas doenças podem ser diagnosticadas pelas alterações dos níveis glicêmicos, que atualmente podem ser mensurados por dois métodos: 0 método laboratorial e o método utilizando glicosímetros portáteis. Portanto, o objetivo desse estudo foi realizar uma análise comparativa da glicose, medida com um glicosímetro portátil de uso humano e outro de uso veterinário, com o método laboratorial, considerado padrão ouro, para avaliar se os equipamentos portáteis podem ser precisos na mensuração glicêmica a campo, em bovinos. Através da veia coccígea foi realizada a coleta sanguínea de 17 bovinos e as amostras foram acondicionadas em tubo com fluoreto de sódio para a análise laboratorial enzimático colorimétrico, e para a aferição da concentração de glicose nos glicosímetros portáteis, foi utilizado 1 gota de sangue. Os dados apresentaram distribuição normal pelo teste de Shapiro-Wilk $(P=0,128)$ e na análise comparativa fornecida pelo teste de Tukey $(P<0,05)$ foram observadas diferenças significativas entre os métodos. Portanto, o glicosímetro humano e o glicosímetro veterinário não podem ser utilizados para a medição da dosagem glicêmica em bovinos, sendo assim, aconselhável fazer somente o uso do método padrão ouro para a mensuração da glicose nessa espécie.
\end{abstract}

Palavras chave: Glicose, metabolismo, método enzimático colorimétrico, ruminantes

\section{Comparative analysis between portable glucometers and laboratory method for the measurement of glycemia in cattle}

\begin{abstract}
Glucose is considered an important source of energy for cells, fetal growth and milk production in ruminants. Some diseases can be diagnosed by changes in blood glucose levels, which can currently be measured by two methods: the laboratory method and the method using portable glucometers. Therefore, the objective of this study was to carry out a comparative analysis of glucose, measured with a portable glucometer for human use and another for veterinary use, with the laboratory method, considered the gold standard, to assess whether portable equipment can be accurate in blood glucose measurement at field, in cattle. Blood was collected from 17 bovines through the coccygeal vein and the samples were placed in a tube with sodium fluoride for colorimetric enzymatic laboratory analysis, and for the measurement of glucose concentration in portable glucometers, 1 drop of blood was used. The data showed normal distribution by the Shapiro-Wilk test $(\mathrm{P}=0.128)$ and in the comparative analysis provided by the Tukey test $(\mathrm{P}<0.05)$ significant differences were observed between the methods. Therefore, the human glucometer and the veterinary glucometer cannot be used to measure the glycemic dosage in cattle, therefore, it is advisable to use only the gold standard method for measuring glucose in this species.
\end{abstract}

Keywords: Glucose, metabolisme, colorimetric enzymatic method, ruminants 


\title{
Análisis comparativo entre glucómetros portátiles y método de laboratorio para medir la glucosa en sangre en bovinos
}

\begin{abstract}
Resumen. La glucosa se considera una fuente importante de energía para las células, el crecimiento fetal y la producción de leche en los rumiantes. Algunas enfermedades pueden diagnosticarse mediante cambios en los niveles de glucosa en sangre, que actualmente se consiguen medir mediante dos métodos: el método de laboratorio y el método que utiliza glucómetros portátiles. Por tanto, el objetivo de este estudio fue realizar un análisis comparativo de glucosa, medida con un glucómetro portátil de uso humano y otro de uso veterinario, con el método de laboratorio, considerado el estándar de oro, para evaluar si los equipos portátiles pueden ser precisos en medición de glucosa en sangre en el campo, para ganado. Se extrajo sangre de 17 bovinos a través de la vena coccígea y las muestras se colocaron en un tubo con fluoruro de sodio para análisis colorimétrico enzimático de laboratorio, y para la medición de la concentración de glucosa en glucómetros portátiles se utilizó 1 gota de sangre. Los datos mostraron distribución normal por la prueba de ShapiroWilk $(\mathrm{P}=0,128)$ y en el análisis comparativo proporcionado por la prueba de Tukey $(\mathrm{P}<$ $0,05)$ se observaron diferencias significativas entre los métodos. Por lo tanto, el glucómetro humano y el glucómetro veterinario no se pueden usar para medir la dosis glucémica en el ganado, por lo tanto, es aconsejable usar solo el método estándar de oro para medir la glucosa en esta especie.
\end{abstract}

Palabras clave: Glucosa, metabolismo, método enzimático colorimétrico, rumiantes

\section{Introdução}

A glicose é considerada uma importante fonte de energia celular, crescimento fetal e produção de leite (Duffield \& LeBlanc, 2009; Hocquette et al., 2006). Dentre vários componentes utilizados para a manutenção do organismo, a concentração de glicose no sangue é um dos fatores mais importantes, e alterações glicêmicas como a hiperglicemia ou hipoglicemia podem revelar informações sobre o estado fisiológico do animal que quando diagnosticadas de forma precoce, podem evitar a morte do paciente (Bush, 2004; Gonzalez \& Silva, 2006).

Das doenças que podem ser diagnosticadas pela alteração dos níveis glicêmicos em ruminantes, podemos citar a enterotoxemia causada pelo Clostridium perfringens tipo D, que causa uma acentuada hiperglicemia. A hipoglicemia pode ser observada em casos de deficiência de cobalto no organismo animal, cetose bovina (Campos et al., 2005) e bezerros que apresentam diarreia (Smith, 2006).

Para avaliar os níveis glicêmicos, atualmente, existem dois sistemas de aferição, o método laboratorial e o glicosímetro portátil (Bromerschenkel et al., 2015; Cordova et al., 2009; Petritz et al., 2013). O método laboratorial é o mais preciso, eficaz e é utilizado como referência e consiste no método colorimétrico enzimático (Gonzalez \& Silva, 2006). Apesar de ser o método padrão ouro, leva mais tempo para a obtenção dos resultados e não é possível fazer a mensuração da concentração glicêmica a campo (Tonyushkina \& Nichols, 2009).

O glicosímetro portátil mede os níveis de glicose automaticamente por aparelho de forma fácil e rápida (Bromerschenkel et al., 2015; Gonzalez \& Silva, 2006). No entanto, foram fabricados e calibrados para fazer a leitura do padrão bioquímico de sangue humano. Para o uso animal, são conhecidos alguns glicosímetros veterinários, porém, são de uso exclusivos para cães e gatos (Petritz et al., 2013). Portanto, o objetivo desse estudo foi realizar uma análise comparativa entre dois glicosímetros portáteis, um de uso humano e o outro de uso veterinário, comparando-os ao método laboratorial, para avaliar se os equipamentos portáteis podem ser precisos para a mensuração glicêmica a campo em vacas de leite, já que, atualmente, não existem glicosímetros específicos para essa espécie.

\section{Material e métodos}

Foi realizada a coleta sanguínea de 17 bovinos independente da raça, sexo, idade e categoria. Esses animais são pertencentes a UEA (Unidade de Ensino e Aprendizado) de bovinocultura de leite, do 
Instituto Federal Catarinense Campus Araquari e a utilização desses animais foi autorizada de acordo com o protocolo 336/2020 do CEUA (Comitê de Ética e Uso de Animais) da instituição.

A coleta de sanguínea foi realizada através da veia coccígea pelo sistema Vacutainer ${ }^{\circledR}$ com agulha $20 x 0,8 \mathrm{~mm}$. Uma gota do sangue foi utilizada para a aferição da concentração glicêmica em cada glicosímetro portátil, sendo um de uso veterinário (WELLION ${ }^{\circledR}$ Gluco Calea) e o outro de uso humano (ACCU-CHEK ${ }^{\circledR}$ Performa). Posteriormente, o sangue coletado foi acondicionado no tubo com fluoreto de sódio.

O glicosímetro veterinário (GV) é acompanhado de três chips codificados para uso exclusivo para as espécies caninas, felinas ou equinas. Para a escolha de qual chip utilizar, foi feita a mensuração glicêmica com uma gota de sangue de um bovino para os três chips. Após dois segundos já era possível realizar a leitura.Visto que, todos eles apresentaram resultados semelhantes, foi optado a utilizar o chip de equinos para avaliar a glicose em bovinos, já que ambas as espécies são animais de grande porte.

As amostras dos tubos com fluoreto de sódio foram encaminhadas para o Laboratório de Patologia Clínica Veterinária do Instituto Federal Catarinense Campus Araquari e centrifugadas (DAIKI 80-2B ${ }^{\circledR}$ ) a $2500 \mathrm{rpm}$ por 15 minutos. Após a obtenção do plasma de cada animal, as amostras foram acondicionadas em eppendorf individuais de $1 \mathrm{~mL}$ e armazenadas em temperatura a $4^{\circ} \mathrm{C}$.

A análise pelo o método enzimático colorimétrico foi realizada de acordo com as instruções de uso do kit reagente (LABTEST Glicose Liquiform Vet ${ }^{\circledR}$ ) que em seguida foi processada em analisador bioquímico (MAX BIO TOUCH ${ }^{\circledR}$ ) semiautomatizado e devidamente calibrado. Cada amostra foi avaliada em duplicata e todos os resultados foram expressos em $\mathrm{mg} / \mathrm{dL}$.

Realizou-se a avaliação da normalidade dos resultados por meio do teste de Shapiro-Wilk e análise de variância. Para a comparação das médias, foi realizado o teste de Tukey. As análises estatísticas foram realizadas no programa $\mathrm{R}$ e o nível de significância adotado foi de 5\%.

\section{Resultados e discussão}

As médias do método laboratorial (ML), do glicosímetro humano $(\mathrm{GH})$ e do glicosímetro veterinário (GV) foram respectivamente, 47,8 mg/dL, 68,7 mg/dL e 86,2 mg/dL e diferiram (P < 0,05). O intervalo entre as análises glicêmicas pelo ML variou entre 36 a $59 \mathrm{mg} / \mathrm{dL}$. No GH, apenas $23,5 \%$ dos resultados estavam dentro do intervalo de referência do ML. No GV, os resultados mostraram uma porcentagem de 94,1\% acima do intervalo do ML (Tabela 1). De acordo com a literatura, os valores de referência para a espécie bovina, variam entre 45 a 75mg/dL (Kaneko et al., 2008). No manual de instruções do kit reagente utilizado para obtenção dos resultados laboratoriais neste estudo, trazem um valor de referência diferente do que é proposto na literatura que varia entre 55 a $95 \mathrm{mg} / \mathrm{dL}$. No ML a média dos valores glicêmicos foi de $47,8 \mathrm{mg} / \mathrm{dL}$ ficando dentro do intervalo de referência proposto na literatura e abaixo do que propôs o manual do reagente.

Em um estudo realizado por Pogliani \& Birgel Junior (2007) com bovinos, foi estabelecido valores de referências glicêmicas de acordo com a faixa etária dos animais. 75,1 a $88,3 \mathrm{mg} / \mathrm{dL}$ para bezerros com até três meses de idade, 64,0 a $76,1 \mathrm{mg} / \mathrm{dL}$ para animais entre três e vinte e quatro meses de idade, 60,6 a 67,2 para vacas adultas com mais de vinte e quatro meses de vida. O intervalo de referência pode diferir pela influência de diversos fatores como a faixa etária, raça, níveis de energia na dieta, produção de leite e condições climáticas. No presente estudo, encontrou-se valores que corroboram com os achados de Pogliani \& Birgel Junior (2007) pois os resultados das concentrações glicêmicas, independente da categoria dos animais, pelo ML variaram entre 36 e $59 \mathrm{mg} / \mathrm{dL}$ estando abaixo de todos os valores referenciais encontrados por esses autores.

A mensuração com o GH apesar de estar dentro do intervalo de referência proposto pela literatura e pelo manual do reagente, diferiu do ML estando em desacordo com o estudo feito por Helayel et al. (2020) que não observaram diferença significativa $(P>0,05)$ entre o glicosímetro portátil humano e o método padrão ouro.

Alguns estudos comparativos entre GH e ML mostraram que os métodos foram eficazes na dosagem glicêmica em cães (Aleixo et al., 2010; Serôdio et al., 2008). Já em gatos (Oliveira et al., 2015) e equinos (Teixeira Neto et al., 2011) foram observadas diferenças entre os métodos. Em bovinos e ovinos, foi 
relato que o glicosímetro portátil e o método enzimático apresentaram diferenças significativas, entretanto os valores finais podem ser reparados a fim de se obter resultados fiáveis para serem usados na clínica (Katsoulos et al., 2011).

Tabela 1. Valores e médias obtidas na determinação da glicose por meio do teste laboratorial e pelos glicosímetros portáteis.

\begin{tabular}{lccc}
\hline & & \multicolumn{2}{c}{ Métodos, ml/dL } \\
\cline { 2 - 4 } Animais & $\begin{array}{c}\text { Laboratorial - Enzimático } \\
\text { colorimétrico }\end{array}$ & Glicosímetro portátil humano & Glicosímetro portátil veterinário \\
\hline 1 & 40 & 89 & 80 \\
2 & 42 & 59 & 87 \\
3 & 50 & 66 & 76 \\
4 & 51 & 63 & 96 \\
5 & 44 & 58 & 98 \\
6 & 49 & 77 & 98 \\
7 & 36 & 59 & 106 \\
8 & 56 & 83 & 67 \\
9 & 52 & 74 & 78 \\
10 & 57 & 79 & 104 \\
11 & 59 & 78 & 105 \\
12 & 48 & 66 & 67 \\
13 & 43 & 64 & 55 \\
14 & 50 & 70 & 85 \\
15 & 54 & 69 & 92 \\
16 & 45 & 69 & 80 \\
Média & 36 & 45 & $86,2^{\mathrm{c}}$ \\
\hline
\end{tabular}

Letras sobrescritas diferentes na linha representam diferença significativa pelo teste de Tukey $(\mathrm{P}<0,05)$.

O glicosímetro de uso veterinário não fornece intervalo de referência para a espécie bovina, visto que, o aparelho é de uso recomendado apenas para caninos, felinos e equinos. A média dos valores obtidos pelo glicosímetro veterinário na espécie bovina foi de $86,2 \mathrm{mg} / \mathrm{dL}$, ficando bem acima da média laboratorial desse estudo e do valor que é proposto pela literatura, porém, estava dentro do intervalo de referência proposto pelo manual do reagente utilizado.

\section{Conclusão}

O glicosímetro humano e o glicosímetro veterinário não são indicados para a aferição da concentração glicêmica em bovinos a campo.

\section{Referências}

Aleixo, G. S., Coelho, M. C. O., Tenório, A. P. M., Guimarães, A. L. N., Andrade, M. B., \& Cavalcanti, H. B. (2010). Uso do glicosímetro portátil para determinar a concentração de glicose no sangue de cães. Ciência Animal Brasileira, 11(3). https://doi.org/10.5216/cab.v11i3.3700.

Bromerschenkel, I., Porfírio, L. C., \& Martins, C. B. (2015). Uso do glicosímetro portátil para a mensuração da glicemia em potros neonatos da raça mangalarga marchador. Acta Veterinaria Brasilica, 9(4), 369-374.

Bush, B. M. (2004). Interpretação de resultados laboratoriais para clínicos de pequenos animais. Editora Roca.

Campos, R., González, F., Coldebella, A., \& Lacerda, L. (2005). Determinação de corpos cetônicos na urina como ferramenta para o diagnóstico rápido de cetose subclínica bovina e relação com a composição do leite. Archives of Veterinary Science, 10(2). https://doi.org/10.5380/avs.v10i2.4413.

Cordova, C. M. M., Valle, J. P., Yamanaka, C. N., \& Cordova, M. M. (2009). Determinação das glicemias capilar e venosa com glicosímetro versus dosagem laboratorial da glicose plasmática. Jornal Brasileiro de Patologia e Medicina Laboratorial, 45(5), 379-384.

Duffield, T. F., \& LeBlanc, S. J. (2009). Interpretation of serum metabolic parameters around the transition period. Southwest Nutrition and Management Conference, 1, 106-114. 
Gonzalez, F. H. D., \& Silva, S. C. (2006). Introdução à bioquímica clínica animal. Gráfica de Universidade Federal do Rio Grande do Sul.

Helayel, M. A., Cunha, N. C., Moron, S. E., Silva, P. C. A. R., Cunha, I. M., Chenard, M. G., Xavier, M., Carvalho, V. de A. N., Nunes, G. S., \& Lopes, S. P. (2020). Comparative analysis between portable glucometer and enzyme method for measurement of blood glucose levels in cattle. Acta Scientiae Veterinariae, 48(1), 1734-1740. https://doi.org/10.22456/1679-9216.102954.

Hocquette, J. F., Sauerwein, H., Higashiyama, Y., Picard, B., \& Abe, H. (2006). Prenatal developmental changes in glucose transporters, intermediary metabolism and hormonal receptors related to the IGF/insulin-glucose axis in the heart and adipose tissue of bovines. Reproduction Nutrition Development, 46(3), 257-272. https://doi.org/10.1051/rnd:2006014

Kaneko, J. J., Harvey, J. W., \& Bruss, M. L. (2008). Clinical biochemistry of domestic animals (6th ed. (ed.); Vol. 1). Academic press.

Katsoulos, P. D., Minas, A., Karatzia, M. A., Pourliotis, K., \& Christodoulopoulos, G. (2011). Evaluation of a portable glucose meter for use in cattle and sheep. Veterinary Clinical Pathology, 40(2), 245-247. https://doi.org/10.1111/j.1939-165X.2011.00309.x.

Oliveira, Y. S. G., Costa Júnior, J. D., Santos-Leonardo, A., \& Morais, K. S. (2015). Comparação entre os métodos laboratorial e portátil na análise da glicemia em felinos com amostras de sangue venoso central e capilar. Ciência Animal Brasileira, 16(2), 279-286.

Petritz, O. A., Antinoff, N., Chen, S., Kass, P. H., \& Paul-Murphy, J. R. (2013). Evaluation of portable blood glucose meters for measurement of blood glucose concentration in ferrets (Mustela putorius furo). Journal of the American Veterinary Medical Association, 242(3), 350-354. https://doi.org/10.2460/javma.242.3.350.

Pogliani, F. C., \& Birgel Junior, E. (2007). Valores de referência do lipidograma de bovinos da raça holandesa, criados no Estado de São Paulo. Brazilian Journal of Veterinary Research and Animal Sciences, 44(05), 373-383.

Serôdio, A. T., Carvalho, C. B., \& Machado, J. A. (2008). Glicemia em cães (Canis familiaris) com glucômetro digital portátil e teste laboratorial convencional. Jornal Brasileiro de Ciência Animal, $1(1), 25-34$.

Smith, M. O. (2006). Tratado de medicina interna de grandes animais (Vol. 1). Manole.

Teixeira Neto, A. R., Botelho, R. G., Sousa, K. E., Gomes, J. L., \& Lima, E. M. M. (2011). Ineficácia do aparelho portátil accutrend plus na clínica médica de eqüinos. ARS Veterinaria, 27, 17-21.

Tonyushkina, K., \& Nichols, J. H. (2009). Glucose meters: a review of technical challenges to obtaining accurate results. Journal of Diabetes Science and Technology, 3(4), 971-980.

Histórico do artigo:

Recebido: 15 de fevereiro de 2021

Aprovado: 21 de março de 2021
Licenciamento: Este artigo é publicado na modalidade Acesso Aberto sob a licença Creative Commons Atribuição 4.0 (CC-BY 4.0), a qual permite uso irrestrito, distribuição, reprodução em qualquer meio, desde que o autor e a fonte sejam devidamente creditados. 